\title{
Setting up the Socialization Solutions for Development of Futsal in Vietnam
}

\author{
Truong Hoai Trung, Ngo Van Hy, Le Quy Phuong \\ Nha Trang University, Ho Chi Minh City University of Sport, Ho Chi Minh City, Viet Nam
}

Email address:

phuongyhtt@gmail.com (Le Q. Phuong)

\section{To cite this article:}

Truong Hoai Trung, Ngo Van Hy, Le Quy Phuong. Setting up the Socialization Solutions for Development of Futsal in Vietnam. International Journal of Sports Science and Physical Education. Vol. 6, No. 2, 2021, pp. 35-40. doi: 10.11648/j.ijsspe.20210602.13

Received: March 21, 2021; Accepted: April 6, 2021; Published: April 16, 2021

\begin{abstract}
In the current trend, the integration process in the economic fields, literature socialization with other countries in the world has been developing strongly. Vietnam's social management has shifted from a central planning mechanism to a market mechanism. The implementation of the policy of socialization in the areas of interest to implement, including sports. Through the development of sport socialization will create conditions for it to develop stronger and more diversified towards service business. Building and developing social and professional organizations in sports will attract a large number of the masses to participate, thereby promoting the resources and creativity of the people in sport development. This researchis conducted with the aim of developing futsal in Vietnam, which is really professional and is the basis for the planning of futsalmanagement in the futsal clubs and training centers on the whole is better in the future. Based on the results of the research on the status of socialization by quantitative and qualitative research methods, we have suggested six groups of solutions consist of Innovation, Capacity Building and Management Effectiveness on; Strengthen the Propagation, Education and Awareness of Development in The professional Environment; Promote the Application of Science and Technology and Sports Medicine in the Training and Organization of Futsal Competitions; Evaluation Results of the Feasibility and Importance of Each Solution in the Solution Group: Step by Step Expand the Futsal Market; Enhance and Improve the Efficiency of Capital Management of Futsal; Develop the Relationship Between the Club and Partners Involved in Futsal. They have been proposed to contribute to improving the socialization of futsal in Vietnam.
\end{abstract}

Keywords: Solutions, Socialization, Futsa, Vietnam

\section{Introduction}

The socialization of sports in Vietnam in general and football in particular has become a policy of practical significance, contributing practical solutions to the difficulties that the State and society are facing. In Vietnam, [1] Futsal is being strongly invested, the local managers of sports are trying to promote and develop the movement for the sports. In 2009, Vietnam hosted the Southeast Asian Futsal Championship, the Asian Indoor Games III, the quarterfinals in the 2014 Futsal Asia Cup, 4th rank in the Futsal Asia in 2016 and the final round of the World cup futsal in 2016. Every year, there are 6 to 8 clubs participating in the national championship and the national cup. Especially, since 2018, Futsal Vietnam will have launched a professional tournament bringing together 6 teams from four cities: Hanoi, Da Nang, Ho Chi Minh City and Can Tho. [5] In this first professional season, the teams are allowed to hire the foreigners to upgrade the league level. These are excellent individuals from three futsal powers: Japan, Uzbekistan and Spain. [4] Foreign players play a role in helping Vietnamese futsal players improve their skills, advanced thinking and competitive edge. [6] The launch of this tournament is expected to be the premise for the goal of setting up a professional futsal playground in the coming years. [9]

However, besides the successes and noteworthy that the Vietnam Futsal still has problems to overcome and must have a long-term strategy to develop in the future. [8] One of the reasons leading to this situation of Futsal in general and in Vietnam is the lack of a specific and long-term planning, the lack of talented young people trained in the systemtic and scientific manner for this sport. [11] In addition, the social work of the organizing committee and of the clubs participating in professional is one of the important issues to be considered. [10] The contents of the funds to organize the 
league, funding activities of the clubs, the work of funding, communication, television rights, transfer players,... are always important factors, affecting the success of the league as well as of each club in the process of operation, which can not ignore the role of socialization in the above contents. [2] Therefore, in order for the socialization of futsal in Vietnam to be effective, the organizers and participating clubs should be able to meet the needs of society and the needs of the audience it is necessary to have realistic solutions to solve outstanding problems and difficulties in the implementation of the socialization of Futsal in Vietnam today. For the reasons mentioned above, the research "Setting solutions for socializing and developing Vietnam Futsal" implemented is necessary to provide scientific basis, objective, useful and practical information, contributing to the development of social work in the development of Futsal in Vietnam to be more effective in the future.

\section{Methods}

The research is aimed to propose solutions for socialization contributing to the development of Futsal in Vietnam.

Use routine research methods to accomplish research objectives including methods of literatrue reviews; Sociological investigation method; SWOT analysis method; Statistical mathematical methods. [7]

Research objects: include 48 professionals in the field of including scientists, sports professionals, managers, coaches, professionals working in the field of futsal, managers, coaches, experts in the professional Futsal, local and foreign Futsal clubs, units and organizations relating to Futsal activities.

\section{Research Results}

Based on the research results of futsal socialization in Vietnam, the research makes SWOT analysis to find the strengths, weaknesses, opportunities and challenges of socialization for the professional. [3] Based on the results of the situation analysis and SWOT analysis, the research team has proposed 06 solutions with 27 specific solutions as detailed in Table 1.

After setting up a system of solutions for socializing and developing Futsal in Vietnam as above, the research has set up the forms of survey and interview experts on the importance and feasibility of the solutions based on the Likert assessment system - 5 levels through 2 surveys.

The Likert scale assesses the importance of solutions including:

(1): Very unimportant; (2): Not important; (3): Important; (4): Quite important; (5): Very important

- Meaning of the average value in the Likert scale used in expert survey:

+ Distance value $=$ Maximum-Minimum value $/ \mathrm{n}=(5-1) / 5$ $=0.8$

+ The meaning of the levels as follows:

1.00 - 1.80: Very unimportant; 1.81 - 2.60: Not important; 2.61 - 3.40: Important

3.41 - 4.20: Quite important; 4.21 - 5.00: Very important

- The Likert scale assesses the feasibility of solutions including:

(1): Very unlikely; (2): Not feasible; (3): feasible; (4): Quite feasible; (5): Very feasible

- Meaning of average value in the Likert scale used in expert survey:

+ Distance value $=$ Maximum-Minimum value $/ \mathrm{n}=(5-1) / 5$ $=0.8$

+ The meaning of the levels as follows:

1.00 - 1.80: Very unlikely; 1.81 - 2.60: Not feasible; 2.61 3.40: feasible; 3.41 - 4.20: Quite feasible; 4.21 - 5.00: Very feasible

Conventions for selecting the solutions in the research: The solutions that are selected only when the average value of interviews is greater than 2.46 as assessed by the experts on the feasibility and importance of solutions.

Table 1. Results for proposing the solutions for socializing and developing Futsal in Vietnam.

\begin{tabular}{|c|c|c|}
\hline No & CONTENTS OF THE SOLUTION & Code \\
\hline \multicolumn{2}{|r|}{ I. Reform, improve the capacity and effectiveness of management } & NCNLHQQL \\
\hline 1 & $\begin{array}{l}\text { Perfect the system of legal documents and professional management documents for activities, focus on the management of } \\
\text { socio-professional organizations operating in the field of; issue regulations on ownership and exploitation of commercial rights } \\
\text { in the field of; Issue regulations on commendation, discipline, handling of violations in the organization of competition, etc. }\end{array}$ & DMNCNLQL1 \\
\hline 2 & $\begin{array}{l}\text { Form social-professional organizations at various levels: the Vietnam Federation - the provincial and city- level Federation - the } \\
\text { district - level Federation (Association) - the club (in communes, wards, towns, villages, residential areas). }\end{array}$ & DMNCNLQL2 \\
\hline 3 & $\begin{array}{l}\text { Form social and professional organizations to protect the interests of athletes, referees and practitioners in the field of (ers' } \\
\text { Association, Referees Association...) }\end{array}$ & DMNCNLQL3 \\
\hline \multicolumn{2}{|r|}{ II. Strengthen the propaganda, education, awareness raising of development in professional environment } & TTGDNCNT \\
\hline 4 & $\begin{array}{l}\text { Strengthen the organization of seminars on the development of professional futsal in order to draw experience, lessons and } \\
\text { unified views, solutions for development of professional futsal in the coming years. }\end{array}$ & TTGDNCNT1 \\
\hline 5 & Focus on promoting the role of television agencies, press agencies and social networks in the development of futsal. & TTGDNCNT2 \\
\hline 6 & $\begin{array}{l}\text { Set up a relationship between the Club and the Fan Club in order to promote the initiative, mobilize resources for the club } \\
\text { development and also orient the activities of the supporters, set up the culture Fan. }\end{array}$ & TTGDNCNT3 \\
\hline \multicolumn{2}{|r|}{ III. Promote the application of science and technology and sports medicine in the training and organization of competitions: } & UDKHCNYH \\
\hline 7 & $\begin{array}{l}\text { Make modernization of training facilities; Equipped with adequate equipment for training, focusing on equipment for analysis, } \\
\text { physical assessment, professional skills, skills and tactical thinking of athletes. }\end{array}$ & UDKHCNYH1 \\
\hline 8 & For club levela, organize a separate sports medicine department for the training. & UDKHCNYH2 \\
\hline 9 & Promote the application of information technology in the management of training and management & UDKHCNYH3 \\
\hline \multicolumn{2}{|r|}{ IV. Group of solutions for gradually expanding the Futsal market } & MRTT \\
\hline 10 & Develop professional clubs (How many professional clubs are suitable for our current conditions) & MRTT1 \\
\hline
\end{tabular}




\begin{tabular}{|c|c|c|}
\hline No & CONTENTS OF THE SOLUTION & Code \\
\hline 11 & Improve tournament quality & MRTT2 \\
\hline 12 & Diversify the form of fee collection and the type of service business & MRTT3 \\
\hline 13 & Strengthen the number of clubs in the local units without developed movement. & MRTT4 \\
\hline \multicolumn{2}{|r|}{$V$. Solutions to enhance and improve the efficiency of capital management of futsal. } & HQQLNV \\
\hline 14 & Strengthen the investment of the central state and provinces, cities and branches & HQQLNV1 \\
\hline 15 & $\begin{array}{l}\text { Find out partners who sign exclusive sponsorship agreements or partial sponsorship for soccer teams, female and male teams. } \\
\text { Diversify forms of advertising, sponsorship based on exploiting the image of the team. }\end{array}$ & HQQLNV2 \\
\hline 16 & $\begin{array}{l}\text { Formulate a comprehensive marketing strategy to enhance the commercial value of the tournaments in the national competition } \\
\text { system and the decent tournaments. }\end{array}$ & HQQLNV3 \\
\hline 17 & $\begin{array}{l}\text { Funding from international organizations: According to FIFA "Target" projects, AFC's "Asian Vision" program, AFF's } \\
\text { "Development Aid", Financial Assistance Program (FAP) of FIFA }\end{array}$ & HQQLNV4 \\
\hline 18 & Broadcast copyright, multimedia copyright, radio advertising... & HQQLNV5 \\
\hline 19 & Make transfer of players & HQQLNV6 \\
\hline 20 & $\begin{array}{l}\text { Strengthen the organization of international exchanges between the national team and the national teams, the strong clubs of } \\
\text { Asia and the world, thereby increasing revenue from advertising activities, talent. sponsorship, events, ticket sales. }\end{array}$ & HQQLNV7 \\
\hline 21 & $\begin{array}{l}\text { Diversify the revenues of professional futsal clubs, focusing on improving the efficiency of facility operations, state-funded } \\
\text { facilities, including activities. }\end{array}$ & HQQLNV8 \\
\hline VI. & lutions for developing relationships between the club and its partners involved in Futsal. & PTMQH \\
\hline 22 & Develop the relationship between the clubs and the state management agencies. & PTMQH1 \\
\hline 23 & Develop relationships between the club and donors & PTMQH2 \\
\hline 24 & Develop the relationship between the club and the media & PTMQH3 \\
\hline 25 & Develop relationships between the club and the fans, the fan club & PTMQH4 \\
\hline 26 & Strengthen the development of international relations and contacts & PTMQH5 \\
\hline 27 & Strengthen the development of media relations and donors... & PTMQH6 \\
\hline
\end{tabular}

\subsection{Results of Experts' Evaluation on the Feasibility and Importance of the Solutions to the Development of Futsal in Vietnam}

The total number of votes cast was 50 , with a response rate of $48(96 \%)$.

Based on the results of the analysis of the average value of the experts' choices, the survey showed that all 6 proposed solutions were satisfactory in terms of importance and feasibility through expert rating (average value of 3.7 to 4.8 ).

In particular, for the most important level: the most valued

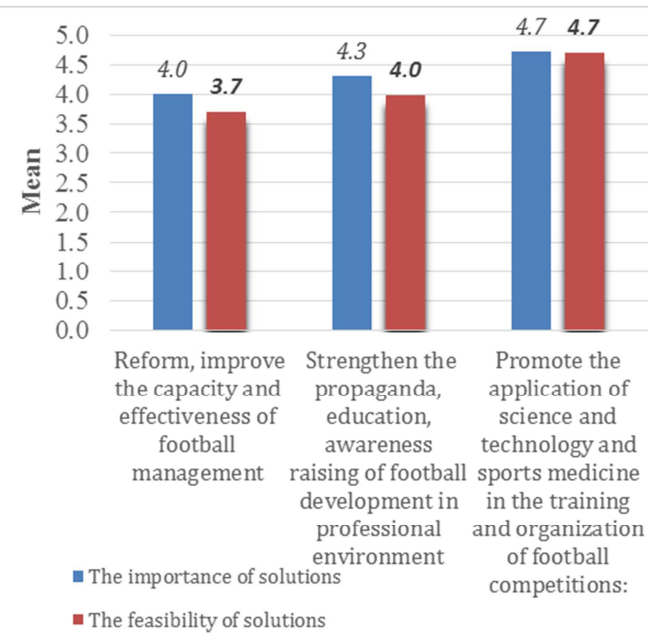

solution group is "Promote the application of science and technology, sports medicine in the training and organization of competition" (average value $=4.7$ ) and the lowest rated group was the "reform, improve capacity, effectiveness of management" (average value $=4.0$ ). For feaseability: The most feasible solution is "Step by step to expand the futsal market" (average value $=4.8$ ) and the lowest evaluated solution group is "reform, improve capacity, effectiveness of management"(average value $=3.7)$. Other results are shown in detail in Figure 1 below.

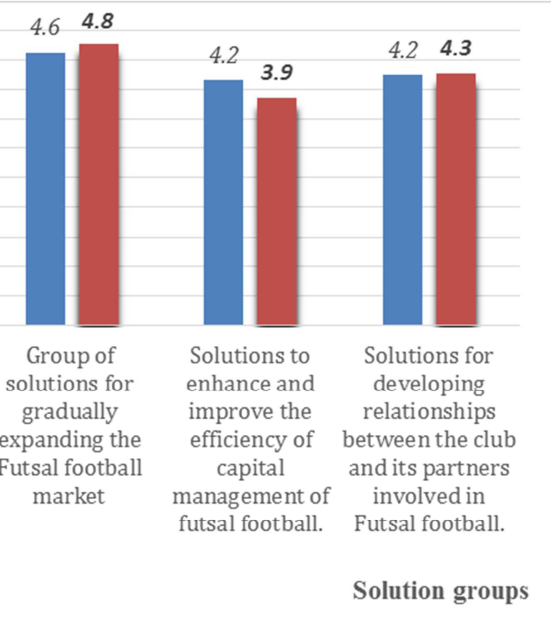

Figure 1. Experts' assessment of the feasibility and importance of futsal development and socialization solutions in Vietnam.

\subsection{Results of Experts' Evaluation on the Feasibility and Importance of Each Solution in the Group of Solutions for Socializing and Developingfutsal in Vietnam}

The system of solutions for socializing and developing futsal in Vietnam will be of high value and reliability when passed by the opinions, evaluations and comments of the experts for the importance and feasibility of each solution. From the analysis results in section 3.1 above. The author analyzes the results of the expert's assessment of the importance and feasibility of each Likert scale solution - five levels. The results include the following: 


\subsubsection{Innovation, Capacity Building and Management Effectiveness on}

There are 3 solutions in the solution group: reform, improve capacity, effectiveness of management. Based on the research results, the experts agreed and highly appreciated the solution "Form social-professional organizations at the various levels: the Vietnam Federation - the provincial - level Federation District-level Association - Clubs (communes, wards, townships, villages, residential areas) (ĐMNCNLQL2). The solution is not appreciated by the experts as to the feasibility of "Form social and professional organizations to protect the interests of athletes, referees and practitioner in the field of (Association of players, Referees Association...) "(ĐMNCNLQL3). Detailed research results are presented in Figure 2.

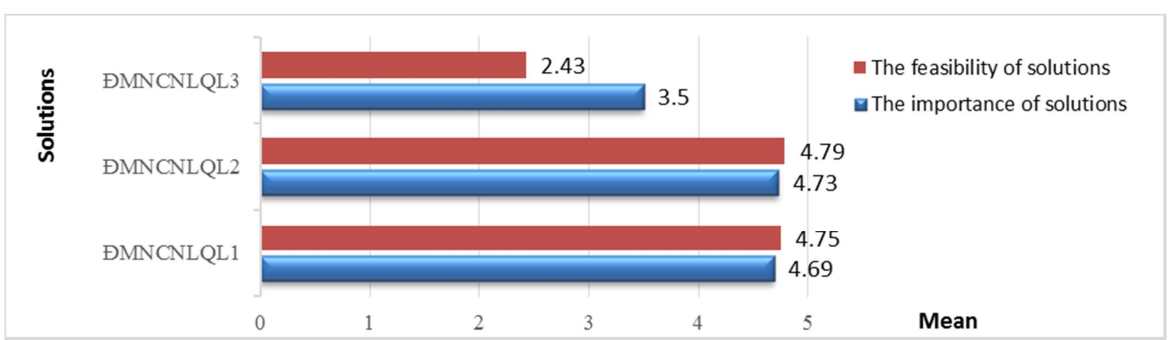

Figure 2. Results of assessment on the feasibility and importance of each solution in the solution group: reform, improve capacity, effectiveness of management.

\subsubsection{Strengthen the Propagation, Education and Awareness of Development in The Professional Environment}

There are 3 solutions in the solution group: Strengthen the propaganda, education, raising awareness of development in professional environment. Based on the results of the research, the solution "Focus on promoting the role of television agencies, press agencies and social networks in the development of futsal" (TTGDNCNT2) is highly appreciated.. In contrast, the solution "Strengthen the organization of seminars on the development of professional futsal to draw experience, lessons and unified views, solutions to develop professional futsal in the coming years. "(TTGDNCNT1) is not highly praised by experts for their feasibility. Detailed research results are presented in Figure 3.

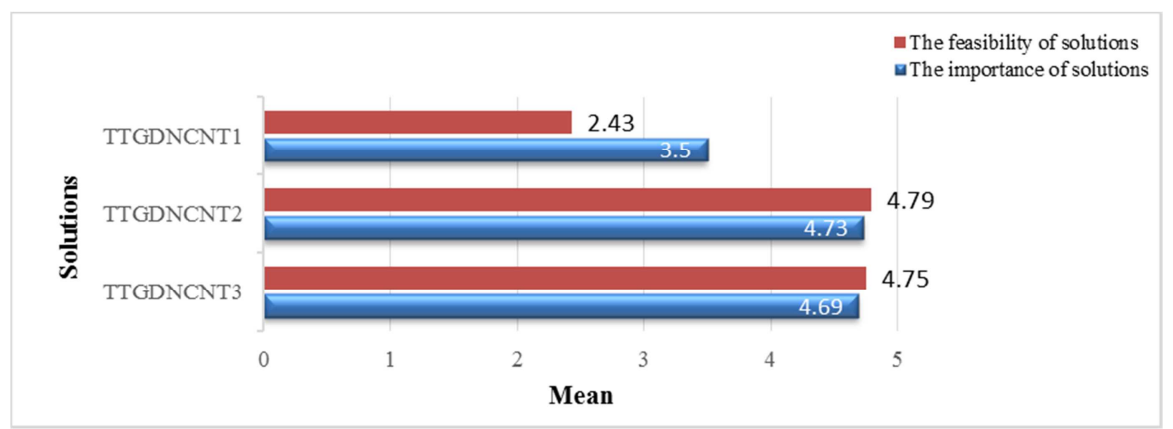

Figure 3. Results of the assessment on the feasibility and importance of each solution in the solution group: Strengthen the propagation, education and awareness of development in the professional environment.

\subsubsection{Promote the Application of Science and Technology and Sports Medicine in the Training and Organization of Futsal Competitions}

There are 3 solutions in the group of solutions: promote the application of science and technology, sports medicine in the training and organization of futsal competitions. The results showed that the solutions were highly appreciated by experts and appreciated the feasibility and importance of the implementation. Other results are shown in Figure 4.

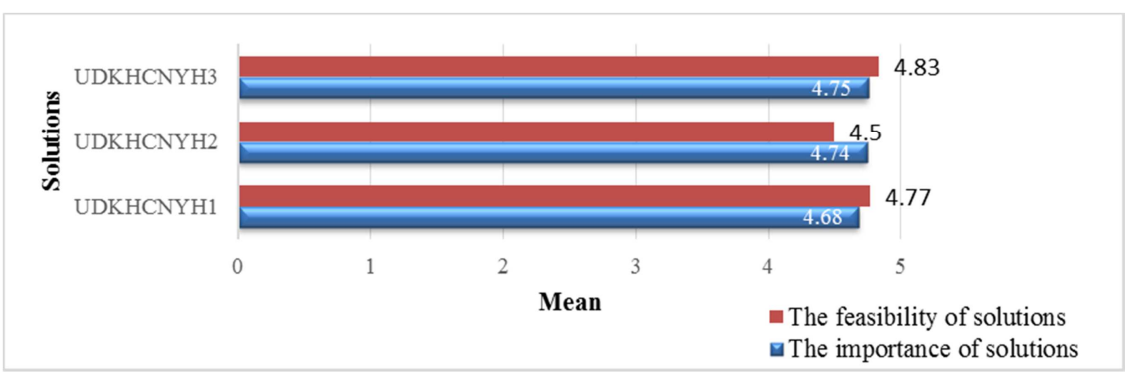

Figure 4. Results of the assessment of the feasibility and importance of each solution in the solution group: promote the application of science and technology, sports medicine in the training and organization of futsal competitions. 


\subsubsection{Evaluation Results of the Feasibility and Importance of Each Solution in the Solution Group: Step by Step Expand the Futsal Market}

Through the research results, the experts unified and highly evaluated the solutions. The most appreciated solution is "Develop professional clubs (How many professional clubs are suitable for our current conditions) (MRTT1). The results of the remaining solutions are presented in Figure 5.

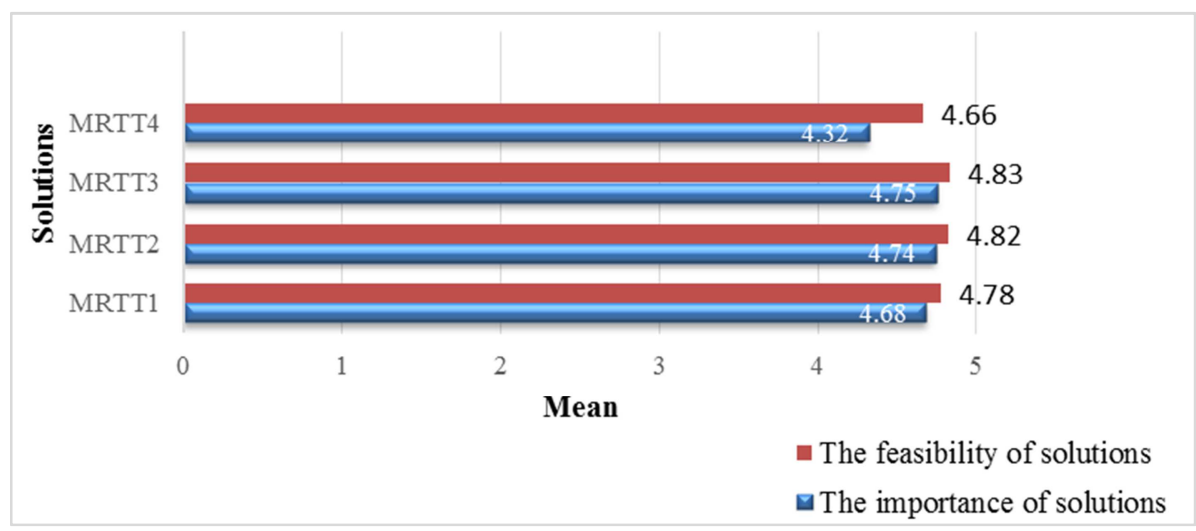

Figure 5. Assessment results of the feasibility and importance of each solution in the solution group: Step by step expand the Futsal market.

\subsubsection{Enhance and Improve the Efficiency of Capital Management of Futsal}

There are 8 solutions in the solution group: Enhance and improve the efficiency of capital management of futsal. Through the research results, the experts agreed and appreciated 05 solutions in turn: "Find out partners to sign exclusive sponsorship contracts or partial sponsorship for teams, young female and male teams. Diversify the forms of advertising, sponsorship based on exploiting the image of the team. "(HQQLNV2)," Form a comprehensive marketing strategy to enhance the commercial value of the tournament in the national competition system and others tournaments ("HQQLNV3"), "Fund from international organizations: FIFA "Target"projects," Asia Vision " AFC, AFF's "Development Aid", FIFA's Financial Aid Program (FESA), "Broadcasting copyright, media copyright, radio advertising. "(HQQLNV5) and" Strengthen the organization of international exchanges between national teams with national teams, strong clubs of Asia and the world through that increased revenue from advertising, sponsorship, events, ticket sales "(HQQLNV7). The remaining solutions are not highly appreciated by the experts. The results are detailed in the Figure below.

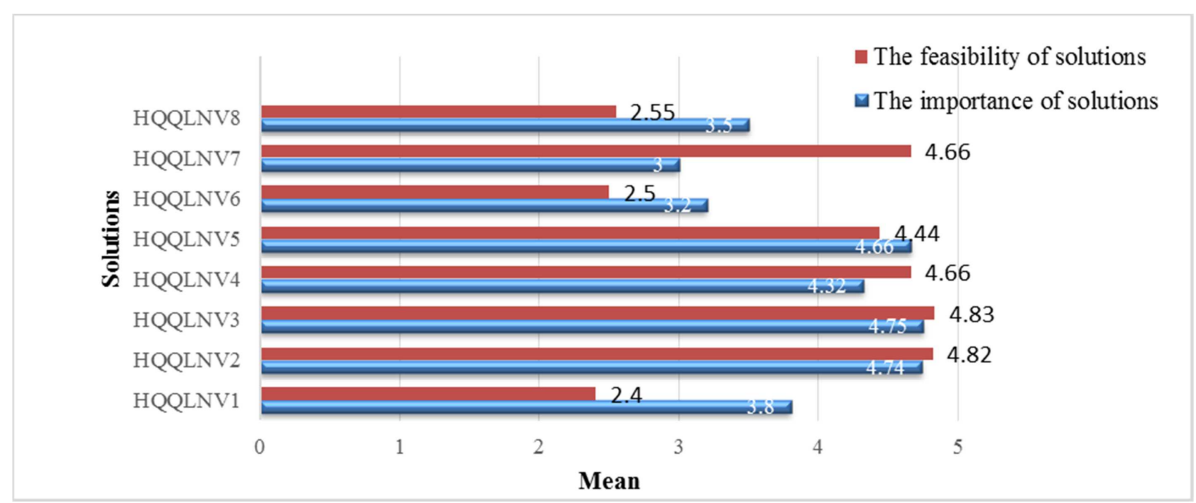

Figure 6. Assessment results of the feasibility and importance of each solution in the solution group. Enhance and improve the effectiveness of the capital management of futsal.

\subsubsection{Develop the Relationship Between the Club and Partners Involved in Futsal}

There are 6 solutions in the solution group" Develop the relationship between the club and the partners involved in futsal. Based on the research results, the solution of "Strengthening communication relations and donors..." (PTMQH6) is not highly appreciated by experts. The remaining solutions are agreed by experts and highly appreciated on the feasibility of implementation. Detailed research results are shown in Figure 7.
Thus, the research has developed six groups of solutions and 21 specific solutions to develop and socializefutsal in Vietnam as follows:

1. Solution: Reform, improve capacity and effectiveness of management, including 2 solutions

2. Solution: promote the propagation, education and awareness of development in professional environment, including 2 solutions

3. Solution: promote the application of science and technology, sports medicine in the training and 
organization of futsal competitions, including 3 solutions

4. Solution: Step by step expand the market Futsal: including 4 solutions

5. Solution: enhance and improve the efficiency of capital management of Futsal: including 5 solutions.

6. Solution: develop relationships between the club and partners involved in Futsal: 5 solutions

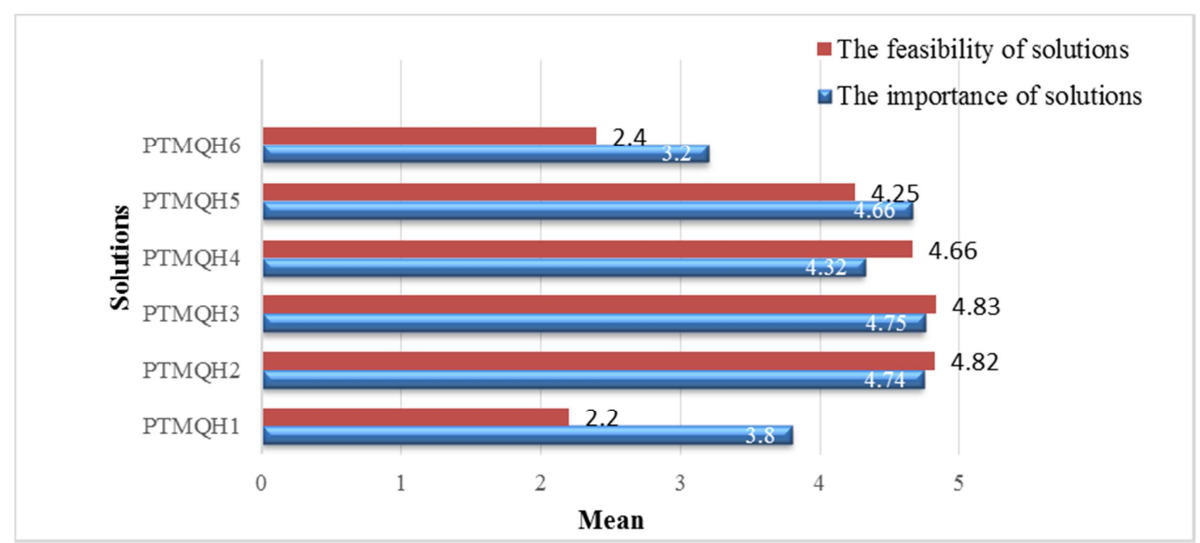

Figure 7. Evaluation results of the feasibility and importance of each solution in the solution group: Develop the relationship between the club and the partners involved in Futsal.

\section{Conclusion}

The results of the research have identified six groups of solutions to contribute to the development of futsal in Vietnam from the perspective of socialization, including the first group of solutions: Reform, improve capacity and effectiveness of management, including 2 solutions, the second group of solutions: promote the propagation, education and awareness of development in professional environment, including 2 solutions; the 3rd group of solutions: promote the application of science and technology, sports medicine in the training and organization of competitions, including 3 solutions; the 4th group of solutions: Step by step expand the market Futsal: including 4 solutions; the 5th group of solutions: enhance and improve the efficiency of capital management of Futsal: including 5 solutions and the 6th group of solutions: develop relationships between the club and partners involved in Futsal: 5 solutions

The result of the research is the objective science which will contribute to orientation for socialization in futsal in the future, as well as feasible when applied in practice in the units.

\section{References}

[1] Le Thiet Can (2016), curriculumof sociology of sport, Publishing House of Vietnam National University Ho Chi Minh City.

[2] Luong Kim Chung (2014), Professional Sports Identification in Vietnam, Scientific Workshop on Solutions for the Development of Vietnam's Sports and Physical Culture in the International Integration Process, HCMC University of Pedagogy. Pg. 70-80.
[3] Dobson, S. and Goddard, J, (2004) "The Economics of ", Cambridge University Press.

[4] Guillaume Bodet and Nicolas Chanavat (2010), Building global brand euity - lessons from the Chinese market, Asia Pacific Journal of Marketing and Logistics, Volume 22, Number 1.

[5] Dang Quoc Nam (2006), Research on Socialization Measures to Exploit Potential for Development of public Sports in Da Nang, doctoral thesis.

[6] Nguyen Trong Nguyen (2017), Research of the system of socialization solutions for professional development in Vietnam ", doctoralthesis on science education.

[7] Le Quy Phuong (2015) Curriculum for method of scientific research in physical education management, Publishing House of Vietnam National University, Ho Chi Minh City.

[8] Stefan SZYMANSKI (2016), Professional Asian Footbal Leagues and the Global Market, Asian Economic Policy Review Vol 11, pp. 16-38.

[9] Huu Thanh (2017), Combination of VBA, futsal Vietnam launches professional tournament for foreigners, http://thethao.sggp.org.vn/ket-hop-vba-futsal-viet-nam-free-lic ense-license-503691.html, accessed 15/03/2018

[10] Nguyen Hoang Thu (2009), "Research on socialization solutions for developing for 3-10 year old children in Nghe An", Doctoral thesis, Hanoi Academy of Sport.

[11] Pham Ngoc Vien (2014), Solutions for Professional Sports Development, Scientific Conference on Solutions for the Development of Vietnam's Sports and Physical Culture in the International Integration Process, HCMC University of Pedagogy. Pg. 69. 